\title{
Differential Relations for the Solutions to the NLS Equation and Their Different Representations
}

\author{
Pierre Gaillard ${ }^{1 *}$
}

\begin{abstract}
Solutions to the focusing nonlinear Schrödinger equation (NLS) of order $N$ depending on $2 N-2$ real parameters in terms of wronskians and Fredholm determinants are given. These solutions give families of quasi-rational solutions to the NLS equation denoted by $v_{N}$ and have been explicitly constructed until order $N=13$. These solutions appear as deformations of the Peregrine breather $P_{N}$ as they can be obtained when all parameters are equal to 0 . These quasi rational solutions can be expressed as a quotient of two polynomials of degree $N(N+1)$ in the variables $x$ and $t$ and the maximum of the modulus of the Peregrine breather of order $N$ is equal to $2 N+1$. Here we give some relations between solutions to this equation. In particular, we present a connection between the modulus of these solutions and the denominator part of their rational expressions. Some relations between numerator and denominator of the Peregrine breather are presented.
\end{abstract}

Keywords: Fredholm determinants, NLS equation, Peregrine breathers, Rogue waves, Wronskians 2010 AMS: 35B05, 35C99, 35Q55, 35L05, 76M99, 78M99

${ }^{1}$ Université de Bourgogne, Dijon, France, ORCID: orcid.org/0000-0002-7073-8284

*Corresponding author: Pierre.Gaillard@u-bourgogne.fr

Received: 25 April 2019, Accepted: 06 August 2019, Available online: 29 December 2019

\section{Introduction}

We consider the one dimensional focusing nonlinear Schrödinger equation (NLS) which can be written in the form

$$
i v_{t}+v_{x x}+2|v|^{2} v=0,
$$

The first results concerning the NLS equation date from the works of Zakharov and Shabat in 1972 who solved it using the inverse scattering method [1, 2]. Its and Kotlyarov first constructed periodic and almost periodic algebro-geometric solutions to the focusing NLS equation in 1976 [3, 4]. Ma found in 1979 the first breather type solution of the NLS equation [5]. In 1983, the first quasi rational solutions of NLS equation were constructed by Peregrine [6]. In 1986, Eleonski, Akhmediev and Kulagin obtained the two-phase almost periodic solution to the NLS equation and got the first higher order analogue of the Peregrine breather $[7,8,9]$. Other analogues of the Peregrine breathers of order 3 and 4 were constructed using Darboux transformations, in a series of articles by Akhmediev et al. [10, 11, 12, 13].

Recently, many works about NLS equation have been published using different methods. We can quote the works of Matveev et al. $[14,15]$ in 2010 for the representation of the solutions in terms of wronskians; those of Gaillard [16, 17, 18] for the solutions given in terms of wronskians and Fredholm determinants, and their quasi-rational solutions limit of order $N$ depending on $2 N-2$ parameters. Akhmediev gave quasi rational solutions using Darboux transformation in several papers [19, 20, 21]. Guo, Ling and Liu in 2012 gave an other representation of the solutions as a ratio of two determinants [22] using generalized Darboux transformation. A new approach has been done by Ohta and Yang in [23] using Hirota bilinear method. Smirnov [24] 
gave solutions with an algebro-geometric approach. Other types of solutions were given by Zhao et al. in [25].

We give some relations between the modulus of these solutions and the denominator part of their rational expression. Some relations between numerator and denominator of the rational solutions are given.

\section{Different representations of solutions to the NLS equation}

\subsection{Solutions of the NLS equation in terms of of Fredholm determinant}

We have to define the following notations.

The terms $\kappa_{v}, \delta_{v}, \gamma_{v}$ and $x_{r, v}$ are functions of the parameters $\lambda_{v}, 1 \leq v \leq 2 N$; they are defined by the formulas :

$$
\begin{aligned}
& \kappa_{v}=2 \sqrt{1-\lambda_{v}^{2}}, \quad \delta_{v}=\kappa_{v} \lambda_{v}, \quad \gamma_{v}=\sqrt{\frac{1-\lambda_{v}}{1+\lambda_{v}}}, \\
& x_{r, v}=(r-1) \ln \frac{\gamma_{v}-i}{\gamma_{v}+i}, \quad r=1,3 .
\end{aligned}
$$

The parameters $-1<\lambda_{v}<1, v=1, \ldots, 2 N$, are real numbers such that

$$
\begin{aligned}
& -1<\lambda_{N+1}<\lambda_{N+2}<\ldots<\lambda_{2 N}<0<\lambda_{N}<\lambda_{N-1}<\ldots<\lambda_{1}<1 \\
& \lambda_{N+j}=-\lambda_{j}, \quad j=1, \ldots, N .
\end{aligned}
$$

The condition (2.2) implies that

$$
\kappa_{j+N}=\kappa_{j}, \quad \delta_{j+N}=-\delta_{j+N}, \quad \gamma_{j+N}=\gamma_{j}^{-1}, \quad x_{r, j+N}=x_{r, j}, \quad j=1, \ldots, N .
$$

Complex numbers $e_{v} 1 \leq v \leq 2 N$ are defined in the following way :

$$
\begin{aligned}
& e_{j}=i \sum_{l=1}^{N-1} a_{l}(j \varepsilon)^{2 l+1}-\sum_{l=1}^{N-1} b_{l}(j \varepsilon)^{2 l+1} \\
& e_{j+N}=i \sum_{l=1}^{N-1} a_{l}(j \varepsilon)^{2 l+1}+\sum_{l=1}^{N-1} b_{l}(j \varepsilon)^{2 l+1} \\
& 1 \leq j \leq N-1
\end{aligned}
$$

$\varepsilon, a_{v}, b_{v}, v=1 \ldots 2 N$ are arbitrary real numbers.

Let $I$ be the unit matrix, and

$$
\varepsilon_{j}=j \quad 1 \leq j \leq N, \quad \varepsilon_{j}=N+j, \quad N+1 \leq j \leq 2 N .
$$

Let's consider the matrix $D_{r}=\left(d_{j k}^{(r)}\right)_{1 \leq j, k \leq 2 N}$ defined by :

$$
d_{v \mu}^{(r)}=(-1)^{\varepsilon_{v}} \prod_{\eta \neq \mu}\left|\frac{\gamma_{\eta}+\gamma_{v}}{\gamma_{\eta}-\gamma_{\mu}}\right| \exp \left(i \kappa_{v} x-2 \delta_{v} t+x_{r, v}+e_{v}\right)
$$

With these notations, the solution to the NLS equation takes the form $[16,17,18]$ :

Theorem 2.1. The function $v$ defined by

$$
v(x, t)=\frac{\operatorname{det}\left(I+D_{3}(x, t)\right)}{\operatorname{det}\left(I+D_{1}(x, t)\right)} e^{2 i t-i \varphi} .
$$

is a solution to the focusing NLS equation depending on $2 N-1$ real parameters $a_{j}, b_{j}, \varepsilon, 1 \leq j \leq N-1$ with the matrix $D_{r}=\left(d_{j k}^{(r)}\right)_{1 \leq j, k \leq 2 N}$ defined by

$$
d_{v \mu}^{(r)}=(-1)^{\varepsilon_{v}} \prod_{\eta \neq \mu}\left|\frac{\gamma_{\eta}+\gamma_{v}}{\gamma_{\eta}-\gamma_{\mu}}\right| \exp \left(i \kappa_{v} x-2 \delta_{v} t+x_{r, v}+e_{v}\right) .
$$

where $\kappa_{v}, \delta_{v}, x_{r, v}, \gamma_{v}, e_{v}$ being defined in(2.1), (2.2) and (2.3). 


\subsection{Wronskian representation}

For this, we need to define the following notations :

$$
\phi_{r, v}=\sin \Theta_{r, v}, \quad 1 \leq v \leq N, \quad \phi_{r, v}=\cos \Theta_{r, v}, \quad N+1 \leq v \leq 2 N, \quad r=1,3,
$$

with the arguments

$$
\Theta_{r, v}=\kappa_{v} x / 2+i \delta_{v} t-i x_{r, v} / 2+\gamma_{v} y-i e_{v} / 2, \quad 1 \leq v \leq 2 N .
$$

The functions $\phi_{r, v}$ are defined by

$$
\phi_{r, v}=\sin \Theta_{r, v}, \quad 1 \leq v \leq N, \quad \phi_{r, v}=\cos \Theta_{r, v}, \quad N+1 \leq v \leq 2 N, \quad r=1,3,
$$

We denote $W_{r}(y)$ the wronskian of the functions $\phi_{r, 1}, \ldots, \phi_{r, 2 N}$ defined by

$$
W_{r}(y)=\operatorname{det}\left[\left(\partial_{y}^{\mu-1} \phi_{r, v}\right)_{v, \mu \in[1, \ldots, 2 N]}\right] .
$$

We consider the matrix $D_{r}=\left(d_{v \mu}\right)_{v, \mu \in[1, \ldots, 2 N]}$ defined in (2.4). Then we have the following statement [17] :

\section{Theorem 2.2.}

$$
\operatorname{det}\left(I+D_{r}\right)=k_{r}(0) \times W_{r}\left(\phi_{r, 1}, \ldots, \phi_{r, 2 N}\right)(0),
$$

where

$$
k_{r}(y)=\frac{2^{2 N} \exp \left(i \sum_{v=1}^{2 N} \Theta_{r, v}\right)}{\prod_{v=2}^{2 N} \prod_{\mu=1}^{v-1}\left(\gamma_{v}-\gamma_{\mu}\right)} .
$$

With these notations, we have the following result [17] :

Theorem 2.3. The function $v$ defined by

$$
v(x, t)=\frac{W_{3}\left(\phi_{3,1}, \ldots, \phi_{3,2 N}\right)(0)}{W_{1}\left(\phi_{1,1}, \ldots, \phi_{1,2 N}\right)(0)} e^{2 i t-i \varphi} .
$$

is a solution to the focusing NLS equation depending on $2 N-1$ real parameters $a_{j}, b_{j}, \varepsilon, 1 \leq j \leq N-1$ with $\phi_{v}^{r}$ defined in (2.5)

$$
\begin{array}{ll}
\phi_{r, v}=\sin \left(\kappa_{v} x / 2+i \delta_{v} t-i x_{r, v} / 2+\gamma_{v} y-i e_{v} / 2\right), & 1 \leq v \leq N \\
\phi_{r, v}=\cos \left(\kappa_{v} x / 2+i \delta_{v} t-i x_{r, v} / 2+\gamma_{v} y-i e_{v} / 2\right), & N+1 \leq v \leq 2 N, \quad r=1,3,
\end{array}
$$

$\kappa_{v}, \delta_{v}, x_{r, v}, \gamma_{v}, e_{v}$ being defined in(2.1), (2.2) and (2.3).

We can give another representation of the solutions to the NLS equation depending only on terms $\gamma_{v}, 1 \leq v \leq 2 N$. From the relations (2.1), we can express the terms $\kappa_{v}, \delta_{v}$ and $x_{r, v}$ in function of $\gamma_{v}$, for $1 \leq v \leq 2 N$ and we obtain :

$$
\begin{aligned}
& \kappa_{j}=\frac{4 \gamma_{j}}{\left(1+\gamma_{j}^{2}\right)}, \quad \delta_{j}=\frac{4 \gamma_{j}\left(1-\gamma_{j}^{2}\right)}{\left(1+\gamma_{j}^{2}\right)^{2}}, \quad x_{r, j}=(r-1) \ln \frac{\gamma_{j}-i}{\gamma_{j}+i}, \quad 1 \leq j \leq N, \\
& \kappa_{j}=\frac{4 \gamma_{j}}{\left(1+\gamma_{j}^{2}\right)}, \quad \delta_{j}=-\frac{4 \gamma_{j}\left(1-\gamma_{j}^{2}\right)}{\left(1+\gamma_{j}^{2}\right)^{2}}, \quad x_{r, j}=(r-1) \ln \frac{\gamma_{j}+i}{\gamma_{j}-i}, \quad N+1 \leq j \leq 2 N .
\end{aligned}
$$

We have the following new representation $[17,26]$ :

Theorem 2.4. The function $v$ defined by

$$
v(x, t)=\frac{\operatorname{det}\left[\left(\partial_{y}^{\mu-1} \tilde{\phi}_{3, v}(0)\right)_{v, \mu \in[1, \ldots, 2 N]}\right]}{\operatorname{det}\left[\left(\partial_{y}^{\mu-1} \tilde{\phi}_{1, v}(0)\right)_{v, \mu \in[1, \ldots, 2 N]}\right]} e^{2 i t-i \varphi}
$$


is a solution to the NLS equation (1.1) depending on $2 N-1$ real parameters $a_{j}, b_{j}, \varepsilon, 1 \leq j \leq N-1$. The functions $\tilde{\phi}_{r, v}$ are defined by

$$
\begin{aligned}
& \tilde{\phi}_{r, j}(y)=\sin \left(\frac{2 \gamma_{j}}{\left(1+\gamma_{j}^{2}\right)} x+i \frac{4 \gamma_{j}\left(1-\gamma_{j}^{2}\right)}{\left(1+\gamma_{j}^{2}\right)^{2}} t-i \frac{(r-1)}{2} \ln \frac{\gamma_{j}-i}{\gamma_{j}+i}+\gamma_{j} y-i e_{j}\right), \\
& \tilde{\phi}_{r, N+j}(y)=\cos \left(\frac{2 \gamma_{j}}{\left(1+\gamma_{j}^{2}\right)} x-i \frac{4 \gamma_{j}\left(1-\gamma_{j}^{2}\right)}{\left(1+\gamma_{j}^{2}\right)^{2}} t+i \frac{(r-1)}{2} \ln \frac{\gamma_{j}-i}{\gamma_{j}+i}+\frac{1}{\gamma_{j}} y-i e_{N+j}\right), \\
& \text { where } \gamma_{j}=\sqrt{\frac{1-\lambda_{j}}{1+\lambda_{j}}}, 1 \leq j \leq N .
\end{aligned}
$$

$\lambda_{j}$ is an arbitrary real parameter such that $0<\lambda_{j}<1, \lambda_{N+j}=-\lambda_{j}, 1 \leq j \leq N$.

The terms $e_{v}$ are defined by (2.3),

where $a_{j}$ and $b_{j}$ are arbitrary real numbers, $1 \leq j \leq N-1$.

Remark 2.1. In the formula (2.6), the determinants $\operatorname{det}\left[\left(\partial_{y}^{\mu-1} f_{v}(0)\right)_{v, \mu \in[1, \ldots, 2 N]}\right]$ are the wronskians of the functions $f_{1}, \ldots, f_{2 N}$ evaluated in $y=0$. In particular $\partial_{y}^{0} f_{v}$ means $f_{v}$.

\subsection{Families of quasi-rational solutions of NLS equation in terms of a quotient of two determinants}

The following notations are used:

$$
\begin{aligned}
& X_{v}=\kappa_{v} x / 2+i \delta_{v} t-i x_{3, v} / 2-i e_{v} / 2, \\
& Y_{v}=\kappa_{v} x / 2+i \delta_{v} t-i x_{1, v} / 2-i e_{v} / 2,
\end{aligned}
$$

for $1 \leq v \leq 2 N$, with $\kappa_{v}, \delta_{v}, x_{r, v}$ defined in (2.1).

Parameters $e_{v}$ are defined by (2.3).

Below the following functions are used :

$$
\begin{aligned}
& \varphi_{4 j+1, k}=\gamma_{k}^{4 j-1} \sin X_{k}, \quad \varphi_{4 j+2, k}=\gamma_{k}^{4 j} \cos X_{k}, \\
& \varphi_{4 j+3, k}=-\gamma_{k}^{4 j+1} \sin X_{k}, \quad \varphi_{4 j+4, k}=-\gamma_{k}^{4 j+2} \cos X_{k},
\end{aligned}
$$

for $1 \leq k \leq N$, and

$$
\begin{aligned}
& \varphi_{4 j+1, N+k}=\gamma_{k}^{2 N-4 j-2} \cos X_{N+k}, \quad \varphi_{4 j+2, N+k}=-\gamma_{k}^{2 N-4 j-3} \sin X_{N+k}, \\
& \varphi_{4 j+3, N+k}=-\gamma_{k}^{2 N-4 j-4} \cos X_{N+k}, \quad \varphi_{4 j+4, N+k}=\gamma_{k}^{2 N-4 j-5} \sin X_{N+k},
\end{aligned}
$$

for $1 \leq k \leq N$.

We define the functions $\psi_{j, k}$ for $1 \leq j \leq 2 N, 1 \leq k \leq 2 N$ in the same way, the term $X_{k}$ is only replaced by $Y_{k}$.

$$
\begin{aligned}
& \psi_{4 j+1, k}=\gamma_{k}^{4 j-1} \sin Y_{k}, \quad \psi_{4 j+2, k}=\gamma_{k}^{4 j} \cos Y_{k}, \\
& \psi_{4 j+3, k}=-\gamma_{k}^{4 j+1} \sin Y_{k}, \quad \psi_{4 j+4, k}=-\gamma_{k}^{4 j+2} \cos Y_{k},
\end{aligned}
$$

for $1 \leq k \leq N$, and

$$
\begin{aligned}
& \psi_{4 j+1, N+k}=\gamma_{k}^{2 N-4 j-2} \cos Y_{N+k}, \quad \psi_{4 j+2, N+k}=-\gamma_{k}^{2 N-4 j-3} \sin Y_{N+k}, \\
& \psi_{4 j+3, N+k}=-\gamma_{k}^{2 N-4 j-4} \cos Y_{N+k}, \quad \psi_{4 j+4, N+k}=\gamma_{k}^{2 N-4 j-5} \sin Y_{N+k},
\end{aligned}
$$

for $1 \leq k \leq N$.

Then we get the following result [26] :

Theorem 2.5. The function $v$ defined by

$$
v(x, t)=\frac{\operatorname{det}\left(\left(n_{j k}\right)_{j, k \in[1,2 N]}\right)}{\operatorname{det}\left(\left(d_{j k)_{j, k \in[1,2 N]}}\right)\right.} e^{2 i t-i \varphi}
$$


is a quasi-rational solution of the NLS equation (1.1) depending on $2 N-2$ real parameters $a_{j}, b_{j}, 1 \leq j \leq N-1$, where

$$
\begin{aligned}
& n_{j 1}=\varphi_{j, 1}(x, t, 0), 1 \leq j \leq 2 N \quad n_{j k}=\frac{\partial^{2 k-2} \varphi_{j, 1}}{\partial \varepsilon^{2 k-2}(x, t, 0),} \\
& n_{j N+1}=\varphi_{j, N+1}(x, t, 0), 1 \leq j \leq 2 N \quad n_{j N+k}=\frac{\partial^{2 k-2} \varphi_{j, N+1}}{\partial \varepsilon^{2 k-2}}(x, t, 0), \\
& d_{j 1}=\psi_{j, 1}(x, t, 0), 1 \leq j \leq 2 N \quad d_{j k}=\frac{\partial^{2 k-2} \psi_{j, 1}}{\partial \varepsilon^{2 k-2}(x, t, 0),} \\
& d_{j N+1}=\psi_{j, N+1}(x, t, 0), 1 \leq j \leq 2 N \quad d_{j N+k}=\frac{\partial^{2 k-2} \psi_{j, N+1}}{\partial \varepsilon^{2 k-2}}(x, t, 0), \\
& 2 \leq k \leq N, 1 \leq j \leq 2 N
\end{aligned}
$$

The functions $\varphi$ and $\psi$ are defined in (2.8),(2.9), (2.10), (2.11).

\section{Structure of the multi-parametric solutions to the NLS equation of order $N$ depending on $2 N-2$ parameters}

\subsection{The quotient of two polynomials of degree $(N(N+1)$ in $x$ and $t$ by an exponential depending on $t$}

Here we present a result which states the structure of the quasi-rational solutions of the NLS equation. It was only conjectured in preceding works $[16,18]$. Moreover we obtain here families of deformations of the $N$ th Peregrine breather depending on $2 N-2$ parameters.

In this section we use the notations defined in the previous sections. The functions $\varphi$ and $\psi$ are defined in (2.8), (2.9), (2.10), (2.11).

The structure of the quasi rational solutions to the NLS equation is given by [27] :

Theorem 3.1. The function $v$ defined by

$$
v(x, t)=\frac{\operatorname{det}\left(\left(n_{j k)_{j, k \in[1,2 N]}}\right)\right.}{\operatorname{det}\left(\left(d_{j k)_{j, k \in[1,2 N]}}\right)\right.} e^{2 i t-i \varphi}
$$

is a quasi-rational solution of the NLS equation (1.1) quotient of two polynomials $R(x, t)$ and $S(x, t)$ depending on $2 N-2$ real parameters $a_{j}$ and $b_{j}, 1 \leq j \leq N-1$.

$R(x, t)$ and $S(x, t)$ are polynomials of degrees $N(N+1)$ in $x$ and $t$.

Remark 3.1. The polynomials $R(x, t)$ and $S(x, t)$ have the same coefficients of degrees $N(N+1)$ in $2 x$ and $4 t$ equal to 1 . The polynomial $B(x, t)$ does not have any real root.

\subsection{The structure of the Peregrine breather of order $n$}

There is any freedom to choose $\gamma_{j}$ in such a way that the conditions on $\lambda_{j}$ are checked. We know from previous works [16, 18] that the (analogue) Peregrine breathers are obtained when all the parameters $a_{j}$ and $b_{j}$ are equal to 0 . In order to get the more simple expressions in the determinants, we choose particular solutions in the previous families.

Here we choose $\gamma_{j}=j \varepsilon$ as simple as possible in order to have the conditions on $\lambda_{j}$ checked, and we have [26, 27] :

Theorem 3.2. The function $v_{0}$ defined by

$$
v_{n, 0}(x, t)=\left(\frac{\operatorname{det}\left(\left(n_{j k}\right)_{j, k \in[1,2 N]}\right)}{\operatorname{det}\left(\left(d_{j k}\right)_{j, k \in[1,2 N]}\right)} e^{2 i t-i \varphi}\right)_{\left(a_{j}=b_{j}=0,1 \leq j \leq N-1\right)}
$$

is the Peregrine breather of order N solution of the NLS equation (1.1) whose highest amplitude in modulus is equal to $2 N+1$.

Remark 3.2. The previous result is given in the frame where the limit of the modulus of the solution when $x$ or t tend to infinity is equal to 1 . We know that if $v(x, t)$ is is a solution to the NLS equation then $u(x, t)=a v\left(a x, a^{2} t\right)$ is also a solution to the NLS equation, for any arbitrary real a.

Remark 3.3. In (3.2), the matrices $\left(n_{j k}\right)_{j, k \in[1,2 N]}$ and $\left(d_{j k}\right)_{j, k \in[1,2 N]}$ are defined in (3.1). 
We have seen in previous section that solutions of NLS equation given by (2.7) can be written in function uniquely of terms $\gamma$. We recall that the terms $\gamma_{j}$ are given by $\gamma_{j}=\sqrt{\frac{1-\lambda_{j}}{1+\lambda_{j}}}, 1 \leq j \leq N ; \lambda_{j}$ is an arbitrary real parameter such that $0<\lambda_{j}<1, \lambda_{N+j}=-\lambda_{j}, 1 \leq j \leq N$.

We can rewrite the result given in (2.7) in a simplest formulation as follows [26, 27] :

Theorem 3.3. The function $v$ defined by

$$
v(x, t)=\frac{\operatorname{det}\left(\left(f_{j k}^{(3)}\right)_{j, k \in[1,2 N]}\right)}{\operatorname{det}\left(\left(f_{j k}^{(1)}\right)_{j, k \in[1,2 N]}\right)} e^{2 i t-i \varphi}
$$

is a quasi-rational solution of the NLS equation (1.1) depending on $2 N-2$ real parameters $a_{j}, b_{j}, 1 \leq j \leq N-1$ where

$$
\begin{aligned}
& f_{j k}^{(r)}=\frac{\partial^{2(k-1)}}{\partial \varepsilon^{2(k-1)}}\left(\gamma^{4 j-1} \sin \left[\frac{2 \gamma}{1+\gamma^{2}} x+4 i \frac{\gamma\left(1-\gamma^{2}\right)}{\left(1+\gamma^{2}\right)^{2}} t-i \frac{r-1}{2} \ln \frac{\gamma-i}{\gamma+i}+\sum_{l=1}^{N-1}\left(a_{l}+i b_{l}\right) \varepsilon^{2 l+1}+(j-1) \frac{\pi}{2}\right]\right)_{(\varepsilon=0)}, \\
& f_{j N+k}^{(r)}=\frac{\partial^{2(k-1)}}{\partial \varepsilon^{2(k-1)}}\left(\gamma^{2 N-4 j-1} \cos \left[\frac{2 \gamma}{1+\gamma^{2}} x-4 i \frac{\gamma\left(1-\gamma^{2}\right)}{\left(1+\gamma^{2}\right)^{2}} t+i \frac{r-1}{2} \ln \frac{\gamma-i}{\gamma+i}+\sum_{l=1}^{N-1}\left(a_{l}-i b_{l}\right) \varepsilon^{2 l+1}+(j-1) \frac{\pi}{2}\right]\right)_{(\varepsilon=0)}, \\
& 1 \leq k \leq N, \quad 1 \leq j \leq 2 N, \quad r \in\{1 ; 3\}, \quad \varepsilon \in] 0 ; 1\left[, \quad \gamma=\varepsilon\left(1-\varepsilon^{2}\right)^{1 / 2} .\right.
\end{aligned}
$$

Remark 3.4. In the previous theorem, the expression $\frac{\partial^{0}}{\partial \varepsilon^{0}} f(x)$ means $f(x)$.

The solution to the NLS equation can be written in the form

$$
v_{N}(x, t)=\frac{R_{N}(x, t)}{S_{N}(x, t)} e^{2 i t}=\left(1+\frac{A_{N}(x, t)}{B_{N}(x, t)}\right) e^{2 i t}
$$

and the Peregrine breather in the form

$$
v_{N, 0}(x, t)=\frac{T_{N}(x, t)}{U_{N}(x, t)} e^{2 i t}=\left(1+\frac{P_{N}(x, t)}{Q_{N}(x, t)}\right) e^{2 i t}
$$

where the index 0 means that all the parameters are equal to 0 .

\section{Differential relation for the NLS equation}

In previous works [26,27], we have proven that the solutions $v_{N}$ to the NLS equation can be written in the form

$$
v_{N}(x, t)=\left(1+\frac{A_{N}(x, t)}{B_{N}(x, t)}\right) e^{2 i t}
$$

We have a very simple relation between the square of the modulus of $v_{N}$ and the denominator part $B_{N}$. This relation appears in a paper of Ling and Zhao [25] where the solutions to the NLS equation are given in the frame of the generalized Darboux transfomation. Here this result and its proof are given in a general frame by the following theorem :

Theorem 4.1. The solutions $v_{N}(x, t)=\left(1+\frac{A_{N}(x, t)}{B_{N}(x, t)}\right) e^{2 i t}$ to the NLS equation verify the following relation

$$
\left|v_{N}(x, t)\right|^{2}=1+\left(\ln B_{N}(x, t)\right)_{x x},
$$

where the subscript $x_{x x}$ means the double derivation with respect to $x$.

Proof. For simplicity with omit the references to $N$ and $(x, t)$ to the solution $v$ and the polynomials $A$ and $B$. If we substitute $v$ by $\left(1+\frac{A}{B} e^{2 i t}\right)$ in the expression $X=i v_{t}+v_{x x}+2|v|^{2} v$, we get

$$
\begin{aligned}
& \frac{2}{B^{3}}\left(|A+B|^{2}(A+B)+A B_{x}^{2}-A B_{x x} B\right) \\
& +\frac{1}{B^{2}}\left(i\left(B A_{t}-A B_{t}\right)-2 B^{2}-2 A B-2 A_{x} B_{x}+A B_{x x}+B A_{x x}\right)=0
\end{aligned}
$$


This can gives the two following relations

$$
|A+B|^{2}(A+B)+(A+B) B_{x}^{2}-(A+B) B_{x x} B-(A+B) B^{2}=0
$$

and

$$
i\left(A_{t} B-A B_{t}\right)+\left(A_{x x} B-2 A_{x} B_{x}+A B_{x x}\right)+\left(B_{x x} B-B_{x}^{2}\right)=0
$$

The first relation can be rewritten as

$$
|A+B|^{2}+B_{x}^{2}-B_{x x} B-B^{2}=0
$$

Then the square of the modulus of $v_{N}$ can be written as

$$
\left|v_{N}\right|^{2}=\frac{|A+B|^{2}}{B^{2}}=1+(\ln B)_{x x},
$$

which proves relation (4.1).

\section{Relations between rational part of the solutions to the NLS equation}

With the preceding notations, we get the following statement

Theorem 5.1. The polynomials of the solutions $v_{N}$ to the NLS equation defined by $(3.3) v_{N}(x, t)=\frac{R_{N}(x, t)}{S_{N}(x, t)} e^{2 i t}$ verify the following relations

$$
\begin{aligned}
& \left(i\left(R_{N}\right)_{t}+\left(R_{N}\right)_{x x}-2 R_{N}\right) S_{N}^{2}-\left(\left(S_{N}\right)_{x x}+i\left(S_{N}\right)_{t}\right) R_{N} S_{N} \\
& -2\left(R_{N}\right)_{x}\left(S_{N}\right)_{x} S_{N}+2\left(\left(S_{N}\right)_{x}^{2}+R_{N} \overline{R_{N}}\right) R_{N}=0 .
\end{aligned}
$$

Proof. It is sufficient to replace in the equation (1.1) $v_{N}(x, t)$ by $\frac{R_{N}(x, t)}{S_{N}(x, t)} e^{2 i t}$.

Proposition 5.1. The coordinates of extrema $\left(x_{0}, t_{0}\right)$ of solutions $v_{N}$ to the NLS equation defined by $(3.3) v_{N}(x, t)=\frac{R_{N}(x, t)}{S_{N}(x, t)} e^{2 i t}$ verify the the following relations

$$
\begin{aligned}
& \left(R_{N}\right)_{x}\left(x_{0}, t_{0}\right) \overline{R_{N}}\left(x_{0}, t_{0}\right) S_{N}\left(x_{0}, t_{0}\right)+\left(\overline{R_{N}}\right)_{x}\left(x_{0}, t_{0}\right) R_{N}\left(x_{0}, t_{0}\right) S_{N}\left(x_{0}, t_{0}\right) \\
& -2\left(S_{N}\right)_{x}\left(x_{0}, t_{0}\right) R_{N}\left(x_{0}, t_{0}\right) \overline{R_{N}}\left(x_{0}, t_{0}\right)=0, \\
& \left(R_{N}\right)_{t}\left(x_{0}, t_{0}\right) \overline{R_{N}}\left(x_{0}, t_{0}\right) S_{N}\left(x_{0}, t_{0}\right)+\left(\overline{R_{N}}\right)_{t}\left(x_{0}, t_{0}\right) R_{N}\left(x_{0}, t_{0}\right) S_{N}\left(x_{0}, t_{0}\right) \\
& -2\left(S_{N}\right)_{t}\left(x_{0}, t_{0}\right) R_{N}\left(x_{0}, t_{0}\right) \overline{R_{N}}\left(x_{0}, t_{0}\right)=0 . \\
& \left(R_{N}\right)_{x}\left(x_{0}, t_{0}\right) S_{N}\left(x_{0}, t_{0}\right)-\left(S_{N}\right)_{x}\left(x_{0}, t_{0}\right) R_{N}\left(x_{0}, t_{0}\right)=0 . \\
& \left(R_{N}\right)_{t}\left(x_{0}, t_{0}\right) S_{N}\left(x_{0}, t_{0}\right)-\left(S_{N}\right)_{t}\left(x_{0}, t_{0}\right) R_{N}\left(x_{0}, t_{0}\right)+2 i S_{N}\left(x_{0}, t_{0}\right) R_{N}\left(x_{0}, t_{0}\right)=0 .
\end{aligned}
$$

where $\bar{a}$ means the complex conjugate of $a$.

Proof. It is sufficient to compute the partial derivatives of (1.1) $v_{N}(x, t)$ defined by $\frac{R_{N}(x, t)}{S_{N}(x, t)} e^{2 i t}$.

Remark 5.1. As a consequence of the result on the highest modulus of the $P_{N}$ breather defined by $(3.4) v_{N, 0}(x, t)=\frac{T_{N}(x, t)}{U_{N}(x, t)} e^{2 i t}$, we get

$$
T_{N}(0,0)=(2 N+1) U_{N}(0,0)
$$




\section{Conclusion}

Different representations of the solutions to the NLS equation have been summarized in this paper, as well as the structure of the quasi rational solutions. Some differential relations have been given in this text for the NLS equation.

From different studies realized by the author, [26]-[32], it seems that the maximums of the modulus of the solutions to the NLS equation are in connection with the zeros of the Yablonski-Vorob'ev polynomials [33, 34].

It would be relevant to study this conjecture.

It would be also relevant to search other types of equations verified by the polynomials $\left(P_{N}, Q_{N}\right),\left(R_{N}, S_{N}\right),\left(A_{N}, B_{N}\right)$ or $\left(T_{N}, U_{N}\right)$.

\section{References}

[1] V. E. Zakharov, Stability of periodic waves of finite amplitude on a surface of a deep fluid, J. Appl. Tech. Phys., 9 (1968), 86-94.

[2] V. E. Zakharov, A. B. Shabat Exact theory of two dimensional selffocusing and one dimensinal self modulation of waves in nonlinear media, Sov. Phys. JETP, 34 (1972), 62-69.

[3] A. R. Its, A. V. Rybin, M. A. Salle, Exact integration of nonlinear Schrödinger equation, Teore. Mat. Fiz., 74 (1988), N. 1, 29-45.

[4] A. R. Its, V. P. Kotlyarov, Explicit expressions for the solutions of nonlinear Schrödinger equation, Dockl. Akad. Nauk. SSSR, S. A, 965(11) (1976).

[5] Y. C. Ma, The perturbed plane-wave solutions of the cubic nonlinear Schrödinger equation, Stud. Appl. Math. 60 (1979), 43-58.

[6] D. H. Peregrine, Water waves, nonlinear Schrödinger equations and their solutions, J. Austral. Math. Soc. Ser. B, 25 (1983), 16-43.

[7] N. Akhmediev, V. Eleonskii, N. Kulagin, Exact first order solutions of the nonlinear Schrödinger equation, Th. Math. Phys., 72(2) (1987), 183-196.

[8] N. Akhmediev, V. Eleonsky, N. Kulagin, Generation of periodic trains of picosecond pulses in an optical fiber: Exact solutions, Sov. Phys. J.E.T.P., 62 (1985), 894-899.

[9] V. Eleonskii, I. Krichever, N. Kulagin, Rational multisoliton solutions to the NLS equation, Soviet Doklady 1986 sect. Math. Phys., 287 (1986), 606-610.

[10] N. Akhmediev, A. Ankiewicz, J. M. Soto-Crespo, Rogue waves and rational solutions of nonlinear Schrödinger equation, Physical Review E,80( 026601) (2009).

[11] N. Akhmediev, A. Ankiewicz, P. A. Clarkson, Rogue waves, rational solutions, the patterns of their zeros and integral relations, J. Phys. A: Math. Theor., 43(122002) (2010), 1-9.

[12] N. Akhmediev, A. Ankiewicz, D. J. Kedziora, Circular rogue wave clusters, Phys. Review E, 84 (2011), 1-7.

[13] A. Chabchoub, H. Hoffmann, M. Onorato, N. Akhmediev, Super rogue waves: Observation of a higher-order breather in water waves, Phys. Review X, 2 (2012), 1-6.

[14] P. Dubard, P. Gaillard, C. Klein, V. B. Matveev, On multi-rogue waves solutions of the NLS equation and positon solutions of the KdV equation, Eur. Phys. J. Special Topics, 185 (2010), 247-258.

[15] P. Dubard, V. B. Matveev, Multi-rogue waves solutions : from the NLS to the KP-I equation, Nonlinearity, 26 (2013), 93-125.

[16] P. Gaillard, Families of quasi-rational solutions of the NLS equation and multi-rogue waves, J. Phys. A: Meth. Theor., 44 (2011), 1-15.

[17] P. Gaillard, Wronskian representation of solutions of the NLS equation and higher Peregrine breathers, Jour. of Math. Sciences: Adv. Appl., 13(2) (2012), 71-153.

[18] P. Gaillard, Degenerate determinant representation of solution of the NLS equation, higher Peregrine breathers and multi-rogue waves, Jour. Of Math. Phys., 54 (2013), 013504-1-32.

[19] D. J. Kedziora, A. Ankiewicz, N. Akhmediev, Triangular rogue wave cascades, Phys. Rev. E, 86(056602) (2012), 1-9.

[20] D. J. Kedziora, A. Ankiewicz, N. Akhmediev, Circular rogue wave clusters, Phys. Review E, 84 (2011), 056611-1-7. 
[21] D. J. Kedziora, A. Ankiewicz, N. Akhmediev, Classifying the hierarchy of the nonlinear Schrödinger equation rogue waves solutions, Phys. Review E, 88 (2013), 013207-1-12.

[22] B. Guo, L. Ling, Q. P. Liu, Nonlinear Schrödinger equation: Generalized Darboux transformation and rogue wave solutions, Phys. Rev. E, 85 (2012), 026607.

[23] Y. Ohta, J. Yang, General high-order rogue waves and their dynamics in the nonlinear Schrödinger equation, Pro. R. Soc. A, 468 (2012), 1716-1740.

[24] A. O. Smirnov, Solution of a nonlinear Schrödinger equation in the form of two phase freak waves, Theor. Math. Phys., 173 (2012), 1403-1416.

[25] L. Ling, L. C. Zhao, Simple determinant representation for rogue waves of the nonlinear Schrödinger equation, Phys. Rev. E, 88 (2013), 043201-1-9.

[26] P. Gaillard, Other 2N-2 parameters solutions to the NLS equation and 2N+1 highest amplitude of the modulus of the N-th order AP breather, J. Phys. A: Math. Theor., 48 (2015), 145203-1-23.

[27] P. Gaillard, Multi-parametric deformations of the Peregrine breather of order N solutions to the NLS equation and multi-rogue waves, Adv. Res., 4 (2015), 346-364.

[28] P. Gaillard, Deformations of third order Peregrine breather solutions of the NLS equation with four parameters, Phys. Rev. E, 88 (2013), 042903-1-9.

[29] P. Gaillard, M. Gastineau, Twenty parameters families of solutions to the NLS equation and the eleventh Peregrine breather, Commun. Theor. Phys, 65 (2016), 136-144.

[30] P. Gaillard, M. Gastineau, Twenty two parameters deformations of the twelfth Peregrine breather solutions to the NLS equation, Adv. Res., 10 (2016), 83-89.

[31] P. Gaillard, Towards a classification of the quasi rational solutions to the NLS equation, Theor. And Math. Phys., 189 (2016), 1440-1449.

[32] P. Gaillard, M. Gastineau Families of deformations of the thirteenth Peregrine breather solutions to the NLS equation depending on twenty four parameters, J. Bas. Appl. Res. Int., 21(3) (2017), 130-139.

[33] A. P. Vorob'ev, On the rational solutions of the second Painlevé equation, Differ. Uravn., 1(1) (1965), 79-81.

[34] A. I. Yablonskii, On rational solutions of the second Painlevé equation, Vesti AN BSSR, Ser. Fiz.-Tech. Nauk, (3) (1959), 30-35. 